\title{
Elaborate Corrective Exercise Program after Surgical Correction for Sprengel's Deformity: A Case Study
}

\author{
Ui-jae Hwang, PT, Ph.D ${ }^{1}$; Sung-hoon Jung, PT, Ph.D ${ }^{1}$; Hyeon-ju Lee, PT, B.H.Sc ${ }^{1}$; \\ Ji-yeon Eun, PT, B.H.Sc' ${ }^{1}$ Moon-hwan Kim, PT, Ph.D ${ }^{2}$ \\ ${ }^{1}$ KOREATECH \& Yonsei R\&D Center, Department of Smart KEMA Station, Seoul, South Korea \\ ${ }^{2}$ Department of Rehabilitation Medicine, Wonju Severance Christian Hospital, Wonju, South Korea
}

Background Sprengel's deformity is a malpositioning anomaly characterized by dysplasia and elevation of the scapula. After Sprengel's deformity corrective surgery, an abnormal scapulohumeral rhythm can be generated because of relative stiffness. An elaborate corrective exercise program is needed to prevent the recurrence of the deformity and maintain the effect of the surgery.

Study design A single-subject case study.

Observations The scapular elevation height and scapular upward rotation angle were measured to confirm the scapulohumeral rhythm during $80^{\circ}$ shoulder abduction using am electromagnetic system in an 8-y-old girl who had undergone Sprengel's deformity corrective surgery 3 y earlier. Shoulder abductor strength were measured using a Smart KEMA strength measurement system, with and without restricted scapular elevation. An elaborate corrective exercise program, including five types of exercises, was prescribed for $6 \mathrm{wk}$. The child's parents were instructed to ensure that scapular elevation was restricted during the exercises. Scapular elevation and upward rotation were measured pre, 2, 4, and $6 \mathrm{wk}$ after an elaborate corrective exercise program. Scapular elevation and upward rotation during $80^{\circ}$ shoulder abduction were significantly decreased, and shoulder abductor strength with restricted scapular elevation was significantly increased $6 \mathrm{wk}$ after the elaborate corrective exercise program.

Conclusions After Sprengel's deformity corrective surgery, an elaborate corrective exercise program is necessary to enable control of scapular elevation during humeral elevation.

Key words Corrective exercise; Sprengel's deformity; Scapulohumeral rhythm; Scapular

JMST

2018; 2(2): 50-57

Published Online

Dec 31, 2018

pISSN 2635-8573

eISSN 2635-8581

Article History

Received 15 September 2018

Revised 19 September 2018

(1st)

Revised 13 October 2018

(2nd)

Accepted 18 October 2018

\section{CONTACT}

agafimu@hanmail.net

Moon-hwan Kim,

Department of Rehabili-

tation Medicine, Wonju

Severance Christian

Hospital, Wonju,

South Korea

This is an Open-Access article distributed under the terms of the Creative Commons Attribution Non-Commercial $\mathrm{Li}$ cense (http://creativecommons. org/licenses/by-nc/4.0) which permits unrestricted non-commercial use, distribution, and reproduction in any medium, proviod the any mework is properly cited. elevation, Scapular upward rotation.

\section{BACKGROUND}

Congenital elevation of the scapula, referred to as Sprengel's deformity, is a complex anomaly of malpositioning and dysplasia of the scapula, with subsequent abnormal development of bone and soft tissue, resulting in muscle hypoplasia and atrophy in the entire shoulder girdle. ${ }^{1}$ Sprengel's deformity leads to cosmetic deformities and is often accompanied by functional impairment. Although many hypotheses have been proposed to explain how this developmental failure occurs, the exact cause remains unknown. ${ }^{1}$

Cavendish reported that the primary aim of surgical treatment in cases of Sprengel's deformity was to improve the appearance and that functional improvements were not guaranteed. ${ }^{2}$ Several surgical procedures have been developed to enhance functional outcomes. ${ }^{3-7}$ One surgical intervention known as Green's method performs placing the scapula in an downward positioned and then transferring inferiorly the muscle attachments of the upper, middle and 
lower trapezius, rhomboids major and minor, and levator scapulae. ${ }^{3-5}$ Woodward procedure was the first to propose the muscle transfers. Since then, several procedures have been suggeted. ${ }^{4,6,7}$

Although various surgical methods have been developed for Sprengel's deformity, there have been no studies of the type of rehabilitation exercise that should be performed to maintain and enhance the postoperative effect. In most previous studies, if the range of motion (ROM) of shoulder abduction was quantitatively restored to the full range after a surgical procedure for Sprengel's deformity, the treatment was judged to be successful and sufficient in terms of functional outcomes. ${ }^{6,8-13}$ However, scapular motion related to the thorax is mainly responsible for the great mobility found in this complex. ${ }^{14}$ Integration of the scapulohumeral rhythm makes the scapula contribute to stable base for glenohumeral movements and mobility to position the arm throughout ROM of shoulder joint. ${ }^{15,16}$ The altered scapular movement affect the normal movement pattern. Therefore, in clinical practice, the scapulohumeral rhythm is used as a shoulder movement quality index of the shoulder complex. ${ }^{17}$

Sahrmann suggested that recruitment patterns changed when a faulty movement pattern was established and that alterations in optimal recruitment could result in a synergistic muscle becoming more dominant than a primary muscle. ${ }^{18}$ As a result, the primary muscle could become weaker, and the synergistic muscle could become stronger. ${ }^{18}$ Prolonged posture deviation could result in an abnormal movement pattern and impaired muscle performance, thereby altering muscle length, joint congruency, and soft tissue flexibility. Thus, following corrective surgery of Sprengel's deformity, qualitative evaluations and elaborate corrective exercise are required.

The present study investigated the effectiveness of an elaborate corrective exercise program on the scapulohumeral rhythm and scapular height during shoulder abduction, in addition to shoulder abductor strength, with and without restricted scapular elevation in a child after corrective surgery for Sprengel's deformity.

\section{CASE PRESENTATION}

An 8-y-old girl presented with complaints of elevation and anterior rotation of her left shoulder, bilateral shoulder asymmetry, and head rotation to the right side. Shoulder abduction and elevation were not limited. The patient lacked horizontal adduction (ROM: $38^{\circ}$ ) and internal rotation (ROM: $60^{\circ}$ ) with scapula stabilization of her left shoulder. No neurological deficits were noted, the patient's gait was normal, and she reported no pain associated with the deformity.

The patient's parents first noticed the deformity when the patient was aged about $3 \mathrm{y}$. The severity of the deformity was assessed based on the four-grade system established by Cavendish $^{2}$ in September 2014, and the patient was diagnosed with Cavendish grade III Sprengel's deformity (moderate: the shoulder joint is elevated $2-5 \mathrm{~cm}$, and the deformity is clearly visible) (Figure 1). In April 2015, the deformity was corrected using a modification of the surgical technique designed by Woodward. After surgery, she was

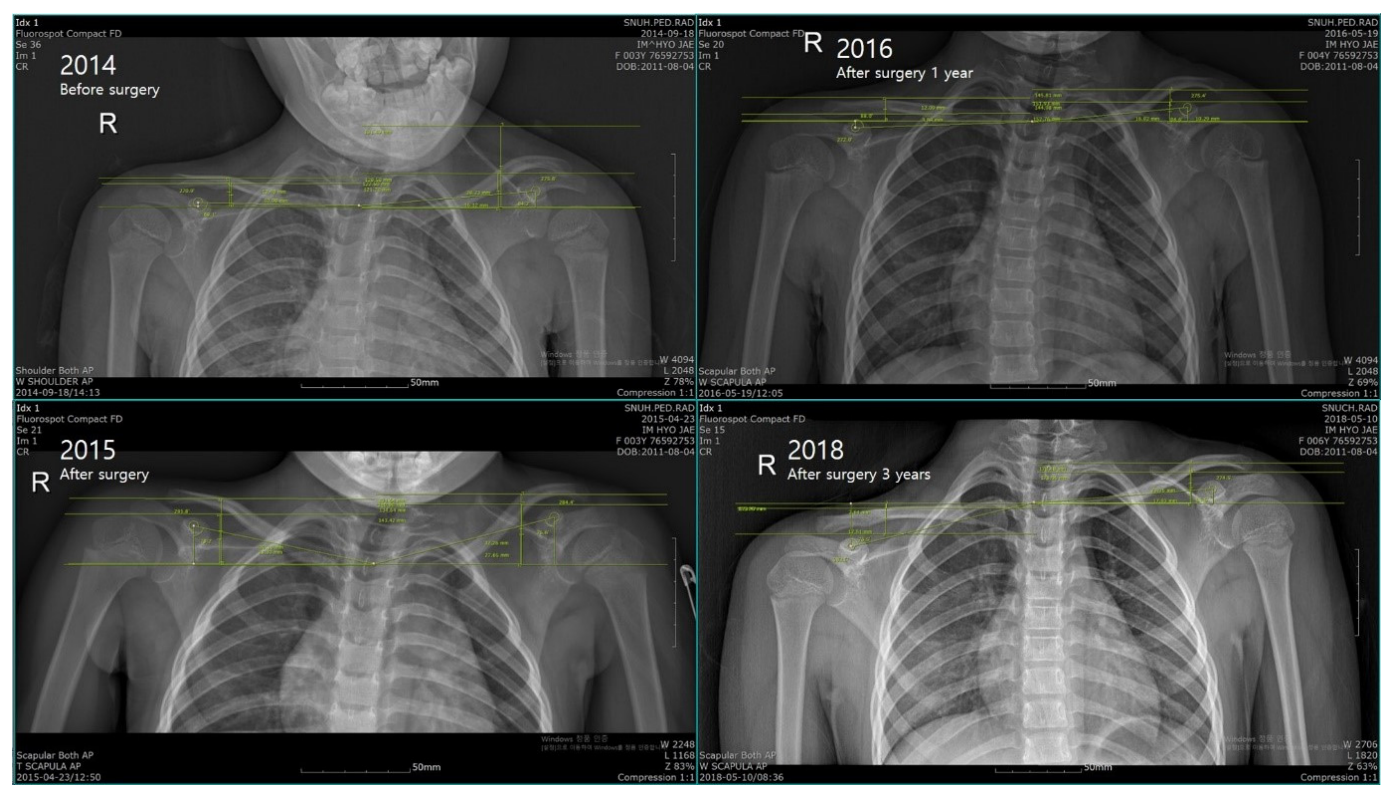

Figure 1. X-ray view of scapular elevation before and after surgery in the 8-y-old patient. 
discharged from the hospital. The patient underwent regular examinations in 2015, 2016 and 2018 (Figure 1).

During the 2 y postoperatively, the patient remained well, with no changes in the appearance of her shoulders. The patient's parents noted re-elevation of her left shoulder as compared with that $2 \mathrm{y}$ earlier. In the last two years, the patient had performed ordinarily activities of daily living. Furthermore, she had continued to swim twice a week for health care during last two years.

\section{INSTRUMENTATION}

The Liberty system (Pholhemus Inc., Colchester, VT, USA), an electromagnetic-based motion analysis system, was used for collecting three-dimensional kinematic data of the scapula. According to the manufacturer, the Liberty system has an accuracy of $0.8 \mathrm{~mm}$ and $0.15^{\circ}$. For measuring the scapulohumeral rhythm and scapular elevation during $80^{\circ}$ shoulder abduction, two sensors were attached to two bony landmarks with adhesive tape: the acromial process of scapula and the lateral epicondyle of the distal humerus. The absolute axes defined by the sensor of the Liberty device were converted to anatomically defined axes derived from digitalized bony landmarks. Raw kinematic data were low-pass filtered at a $6-\mathrm{Hz}$ cutoff frequency and converted into anatomically defined rotations. ${ }^{19,20}$

A Smart KEMA strength measurement system (KOREATECH Co., Ltd., Seoul, Korea) was used to measure isometric shoulder abductor strength, with and without restricted scapular elevation $(3 \mathrm{kgf}){ }^{21}$ The force signals were measured to determine isometric shoulder abductor strength, and the data were transferred to a tablet (Galaxy tab A6 10.1, Samsung Inc., Seoul, Korea) via a Bluetooth connection and analyzed using Smart KEMA application software (KOREATECH Co., Ltd.).

\section{EXPERIMENTAL PROCEDURE}

\section{Pre- and post-tests}

Pre- and post-tests were administered before and 2, 4, and $6 \mathrm{wk}$ after the elaborate corrective exercise program. To evaluate the quality of shoulder movement and assess scapulohumeral rhythm, the scapular upward rotation angle and scapular elevation height were measured in $80^{\circ}$ shoulder abduction. Muscle strength was also evaluated in shoulder abductor strength, with and without restricted scapular elevation.

1) Scapulohumeral rhythm: Scapular upward rotation and scapular elevation height
Before testing the scapulohumeral rhythm, the patient was asked to perform full active abduction ROM in the frontal plane three times. The raw kinematic data were lowpass filtered at a $6-\mathrm{Hz}$ cutoff frequency and converted into anatomically defined rotations. The scapular rotation angle relative to a reference was described as rotation about $X$ (anterior tipping/posterior tipping), Z (medial rotation/lateral rotation), and Y (upward rotation/downward rotation). The scapular upward rotation angle and height of the scapular elevation were measured in the $\mathrm{Y}$ and $\mathrm{Z}$ axes, The kinematics data during $80^{\circ}$ shoulder abduction in the frontal plane were collected before and 2, 4, and $6 \mathrm{wk}$ after the elaborate corrective exercise program. The changes in kinematics were calculated for data analysis. The high reliability (ICC $[2, \mathrm{k}]=0.91-0.99)$ and accuracy (less than $1.2^{\circ}$ upward rotation standard error of measurement) of this approach have been described previously. ${ }^{19,20}$

2) Shoulder abductor strength, with and without restricted scapular elevation

Submaximal isometric contraction of shoulder abduction was performed twice before each test. Isometric strength measurements were performed twice for $5 \mathrm{~s}$ under two conditions: (1) shoulder abduction and (2) shoulder abduction with restricted scapular elevation.

The strength of the shoulder abductor without restricted scapular elevation was measured at $80^{\circ}$ during shoulder abduction in the frontal plane. A belt was attached to the distal humerus of the patient. The tension of the belt was controlled, with shoulder abduction at $80^{\circ}$. To measure the maximum isometric force of shoulder abduction, the patient performed shoulder abduction while pulling the belt at maximum effort for $5 \mathrm{~s}$, without moving her body. ${ }^{21}$ The value was calculated as the average of the values for $3 \mathrm{~s}$ among the $5 \mathrm{~s}$. The mean values of two trials were used for data analysis. To avoid muscle fatigue, the patient rested for $30 \mathrm{~s}$ after each trial and for $2 \mathrm{~min}$ among conditions. ${ }^{21}$

In addition, the strength of the shoulder abductor with restricted scapular elevation was measured at $80^{\circ}$ during shoulder abduction in the frontal plane and restricted elevation of the patient's scapula in manual stabilization. The strength measurements with and without restricted scapular elevation were performed in the same way.

\section{Elaborate corrective exercise program}

The child's parents received education regarding the requirements of the elaborate corrective exercise program. The program was implemented for $6 \mathrm{wk}$, with five sessions per week. Once a week, her parents were instructed to 
ensure that the child exercised under the guidance of a physical therapist in a KEMA station. When the child performed the exercise program at home, her parents supervised her to ensure that the exercises were performed correctly. In terms of the composition of the exercise program, it involved maintaining a controlled scapular position without scapular elevation during a combination of five exercises (cross-body stretching, levator scapulae stretching, upper trapezius stretching, scapular protraction exercise, and scapular posterior tilt exercise). When performing the exercises at home, the child's parents confirmed that the scapula was moving up by palpating the superior angle of the scapula with their hands. If scapular elevation occurred, the child's parents stopped the exercise and advised the child that her shoulder bone had gone up. The child then performed the exercise again. Each exercise position was maintained for $10 \mathrm{~s}$, and the exercise was repeated 10 times.

\section{1) Cross-body stretching}

Cross-body stretching was performed in a supine position. One of the parents held the child's scapula by grasping the acromion and lateral border of the scapula. ${ }^{22}$ The child then flexed her left shoulder and elbow to $90^{\circ}$. She used her right hand to grasp her left elbow (stretching side) and then pulled her elbow of the left side to the right side on a transverse plane, with a relaxed stretching arm as far as possible. This position was maintained for $10 \mathrm{~s}$.

\section{2) Levator scapulae stretching}

The child lay in a prone position, with her head contralaterally rotated to the left side. She held her head and then pulled it in cervical flexion with her right hand and abducted her left shoulder as much as possible, without scapular elevation. One of her parents then applied an inferolateral to the superior angle of the scapula on the left side to restrict scapular elevation and permit scapular upward rotation.

\section{3) Upper trapezius stretching}

The child sat upright on a chair and looked straight ahead. To reduce scapular elevation and upward rotation, she placed her left hand below her buttocks. The child's parent held the child's scapula by grasping the acromion and lateral border of the scapula. She was then asked to perform deep neck flexion, ipsilateral neck rotation toward the left side and right side-bending using the opposite hand in a diagonal direction. The child maintained deep neck flexion while performing the left neck rotation and right side-bending during stretching. ${ }^{23}$

\section{4) Scapular protraction exercise}

The child was placed in a supine position. When her left shoulder was in $90^{\circ}$ flexion, her elbow was fully extended. Thus, the scapula was in a neutral position. This position approximates the normal anatomical position. She then extended her left elbow during exertion of maximal force in the forward direction. One of the parents held the child's scapula by grasping the acromion to restrict scapular elevation and the rib cage to control trunk rotation. ${ }^{24}$

\section{5) Scapular posterior tilt exercise}

The child was placed in the prone position, with the humerus diagonally overhead, shoulder abduction of 130 $145^{\circ}$, and her forearm in a neutral position. ${ }^{25}$ The child was then asked to place her right hand under her forehead and lay slightly on the forehead with the dorsum of her hand. While she lifted her left arm, with her elbow extended until her tragus, her parent restricted scapular elevation by pushing the superior angle of the scapula.

\section{RESULTS}

To determine the effects of the exercise program on the patient, the minimal detectable change (MDC) was calculated for scapular upward rotation and scapular elevation height during $80^{\circ}$ shoulder abduction and for shoulder abductor strength, with and without restricted scapular elevation. ${ }^{26}$ The MDC was calculated using the standard error of measurement (SEM), which indicated the accuracy of the measurement. It was computed using the following equation: $\mathrm{SEM}=\mathrm{SD} \sqrt{(1-I C C)}$, where $\mathrm{SD}$ and $\mathrm{ICC}$ are the standard deviation and intraclass correlation coefficient, respectively. ${ }^{26}$ The formula to obtain $\mathrm{MDC}$ at the $95 \%$ confidence level $\left(\mathrm{MDC}_{95}\right)$ was $\mathrm{MDC}=1.96 \times \sqrt{2} \times S E M$. The pre- and post-program results were compared and deemed significant if they differed by an MDC value or more. ${ }^{26}$ The measured values and MDC values for each variable are shown in Table 1, Table 2 and Figure 2.

\section{DISCUSSION}

The main finding in the present case was the detection of an abnormal scapulohumeral rhythm after Sprengel's deformity correction surgery. In addition, the elaborate corrective exercise program in which scapular elevation was controlled significantly decreased scapular elevation by generating an abnormal scapulohumeral rhythm during shoulder abduction. There were significance of declination for scapular elevation after 2, 4 and $6 \mathrm{wk}$ and scapular 
Table 1 . The results of scapular motion during $80^{\circ}$ shoulder abduction before and after the exercise program

\begin{tabular}{cccccc}
\hline \multicolumn{1}{c}{ Variables } & \multicolumn{2}{c}{ Kinematics } & ICC (95\% CI) & SEM & MDC95 \\
\hline & Pre-exercise & 27.49 & & & 1.25 \\
Scapular upward & After 2 wk & 27.08 & 0.71 & 0.45 & 0.12 \\
rotation angle $\left(^{\circ}\right)$ & After 4 wk & $25.26^{*}$ & $(-10.40,0.99)$ & & 0.04 \\
& After 6 wk & $17.05^{*}$ & & \\
Height of scapular & Pre-exercise & 7.57 & & & \\
elevation (cm) & After 2 wk & $6.49^{*}$ & $(-2.56,0.99)$ & & \\
& After 4 wk & $5.30^{*}$ & & & \\
\hline
\end{tabular}

*changing value (after - pre value) $>$ MDC

Table 2. The results of shoulder abductor strength, with and without restricted scapular elevation before and after the exercise program

\begin{tabular}{cccccc}
\hline \multicolumn{1}{c}{ Variables } & \multicolumn{1}{c}{ Strength } & ICC (95\%CI) & SEM & MDC95 \\
\hline \multirow{2}{*}{$\begin{array}{c}\text { Shoulder abductor strength } \\
\text { without restricted scapular }\end{array}$} & Pre-exercise & 2.8 & & & 0.23 \\
elevation (kgf) & After 2 wk & 2.9 & 0.94 & & \\
& After 4 wk & 2.9 & $(-1.51,0.99)$ & & 0.08 \\
After 6 wk & 2.8 & & 0.05 \\
Shoulder abductor strength & Pre-exercise & 1.4 & & \\
with restricted scapular & After 2 wk & 1.4 & $(-3.33,0.99)$ & & \\
elevation (kgf) & After 4 wk & $1.6^{*}$ & & & \\
& After 6 wk & $2.0^{*}$ & & & \\
\hline
\end{tabular}

${ }^{*}$ changing value (after - pre value) $>$ MDC

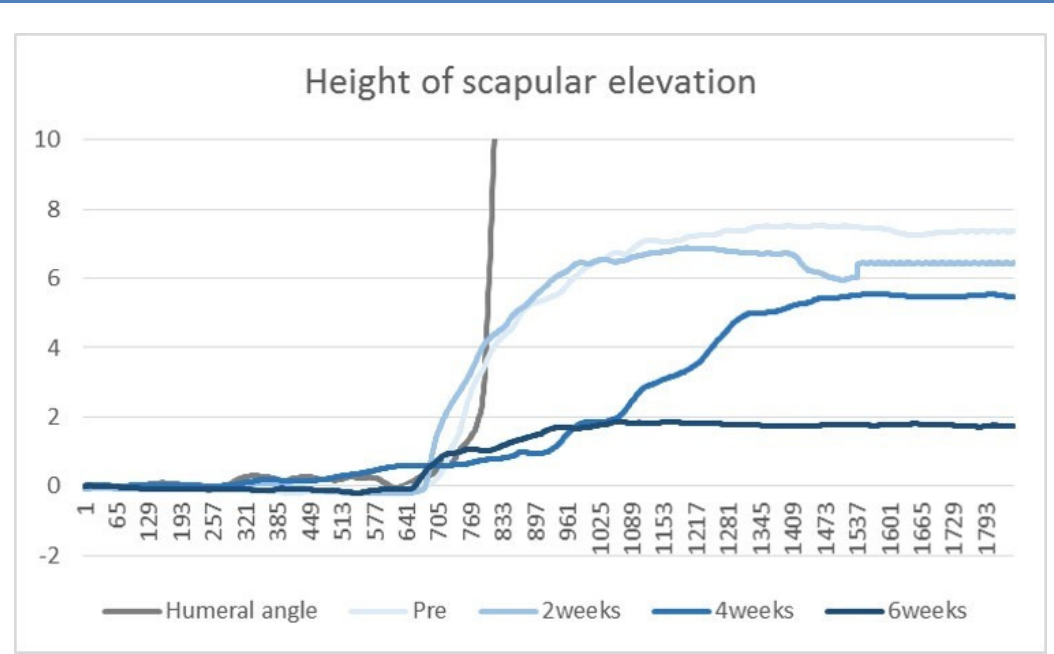

Figure 2. Height of scapular elevation kinematics during shoulder abduction before and after the exercise program.

upward rotation after 4 and $6 \mathrm{wk}$, confirming that changing values (after-pre value) exceed the MDC values. Furthermore, shoulder abductor strength with restricted scapular elevation significantly increased following the elaborate corrective exercise program finding that changing values (after-pre value) after 4 and 6 wk exceed the MDC value.

Shoulder abduction is a habitual movement in functional activities. ${ }^{27}$ As shown in the present case, performing an 
elaborate corrective exercise program after Sprengel's deformity correction surgery could prevent the appearance of a second generation of scapular because of exposure to repetitive habitual shoulder abduction movements.

Sahrmann proposed that subjects with upper trapezius pain have excessive scapular elevation during shoulder abduction or flexion to $90^{\circ}$ could be a possible reason for pain by overload. ${ }^{18}$ The upper trapezius and levator scapulae are scapular elevators. In Woodward surgical procedures, the subcutaneous tissue is undermined to display the vertebral border of the scapula and the orientation of the trapezius muscle fibers. ${ }^{9}$ The origin of the trapezius is then detached from the spinous processes. After removing the trapezius muscle sheet from the spinous processes, the omovertebral bone or fibrous bands of levator scapulae attached to the superior border of the scapula is exposed. ${ }^{9}$ The omovertebral bone is completely removed from its attachment to the spinous process of the cervical spine and superior angle of the scapula. After removal of the omovertebral bone and the release of the levator scapulae muscles, the scapula is free, and its mobility is similar to that of the opposite normal scapula. ${ }^{9}$ The tough fibrous origins of the trapezius and rhomboid muscles are then reattached from the proximal to distal positions. ${ }^{9}$

In the process of recovery after surgery, the excised muscle fibers of the levator scapulae and upper trapezius muscle result in decreased elasticity of collagen fibers. This can lead to relative muscle stiffness as compared with that of the other scapular muscles and to an abnormal scapulahumeral rhythm. ${ }^{24}$ In the present case, repetitive overhead shoulder movement during swimming could have accelerated scapular elevation and the generation of an abnormal scapulohumeral rhythm. ${ }^{20}$ Scapular elevators (i.e., the upper trapezius and levator scapulae) may be overused in repetitive overhead shoulder movements and result in increased muscle tension toward upward and elevation.

Forte et al., suggested that the scapular upward rotation angle was $18.2-23.5^{\circ}$ during $80^{\circ}$ shoulder abduction in healthy individuals. ${ }^{28}$ In the present case, the scapular upward rotation angle was $27.49^{\circ}$ during $80^{\circ}$ shoulder abduction. After the 4- and 6-wk elaborate corrective exercise program, scapular elevation and upward rotation decreased significantly, respectively, as compared with the baseline values. There are several possible explanations for the decrease in scapular elevation and upward rotation. First, upper trapezius and levator scapulae stretching included in the elaborate corrective exercise program could have decreased muscle tension toward upward. Excessive upper trapezius and levator scapulae contraction can cause scapular elevation and upward rotation. ${ }^{18,29}$ Second, bio- feedback of scapular elevation during the elaborate corrective exercise program by confirming her parents could have affected motor control during scapular motion and shoulder abduction. Third, the scapular protraction and posterior tilt exercise could have activated scapular stabilizers (i.e., serratus anterior and lower trapezius muscles). ${ }^{24}$ The main muscles thought to facilitate scapular posterior tilt are the lower trapezius and serratus anterior. ${ }^{30}$ Janda described muscle imbalances as impaired relation-ships between muscles (upper trapezius and levator scapulae) prone to tightness that lose extensibility and muscles (lower trapezius and serratus anterior) prone to inhibition and weakness. $^{31}$ Thus, serratus anterior and lower trapezius strengthening could affect muscle imbalance.

In the present case, shoulder abductor strength with restricted scapular elevation was significantly increased after the 4- and 6-wk elaborate corrective exercise program. The cross-body stretching included in the elaborate corrective exercise program could have increased the flexibility of the glenohumeral joint by stretching the posterior deltoid muscle and posterior joint capsules. ${ }^{22}$ Due to the limited glenohumeral joint motion, scapulothoracic joint movements were more dominant than glenohumeral joint movements. ${ }^{18}$ Examples of scapular compensatory motions are excessive elevation instead of glenohumeral joint flexion or abduction. By causing abnormalities in coordinated scapular rotation, changes in dominance among the scapular rotators could alter the muscle balance of the scapula. ${ }^{32}$ Thus, recruitment of the shoulder abductors could increase without scapular elevation and upward rotation because of increased glenohumeral joint flexibility.

The present study had several limitations. The effectiveness of an exercise may be reduced if it is conducted with insufficient voluntary contraction. This was a possibility in the current study because the exercise program incorporated isometric contraction with voluntary contraction as resistance.

\section{CONCLUSION}

This study confirmed the presence of an abnormal scapulohumeral rhythm during shoulder abduction in an 8y-old child after Sprengel's deformity corrective surgery and investigated the effect of a 6-wk elaborate corrective exercise program to restrict and confirm the movement of scapular elevation. Generally, after Sprengel's deformity corrective surgery, a 4-wk period is required to maintain fixation of the shoulder, and then passive and active shoulder abduction exercises are performed. Due to the relative stiffness caused by the abnormal scapulohumeral 
rhythm generated after Sprengel's deformity corrective surgery, an elaborate corrective exercise program is required postsurgery to be able to control and regulate scapular elevation during shoulder abduction. Further studies on the scapulohumeral rhythm during shoulder abduction in various humeral elevation angles are required in patients following Sprengel's deformity corrective surgery.

\section{Key Points}

Question What type of rehabilitation exercise is required after Sprengel's deformity correction surgery?

Findings After Sprengel's deformity correction surgery, scapular elevation can recur because of an abnormal scapulahumeral rhythm.

During shoulder abduction, excessive scapular elevation and upward rotation decreased following an elaborate corrective exercise program.

Meaning After Sprengel's deformity correction surgery, elaborate corrective exercise should be applied to educate normal scapulohumeral rhythm and to prevent recurrence.

\section{Article information}

Conflict of Interest Disclosures: None.

Funding/Support: None.

Acknowledgment: None.

\section{REFERENCES}

1. Jeannopoulos CL. Congenital elevation of the scapula. JBJS. 1952;34(4):883-892.

2. Cavendish M. Congenital elevation of the scapula. The Journal of Bone and Joint Surgery British Volume. 1972; 54(3):395-408.

3. Beaty JH. Congenital anomalies of trunk and upper extremity. Campbell'S Operative Orthopaedics, 10th edn CV Mosby, St Louis. 2003:1125-1140.

4. Leibovic SJ, Ehrlich M, Zaleske D. Sprengel deformity. The Journal of Bone and Joint Surgery American Volume. 1990;72(2):192-197.

5. Woodward JW. Congenital elevation of the scapula: Correction by release and transplantation of muscle origins. JBJS. 1961;43(2):219-228.

6. Borges JLP, Shah A, Torres BC, Bowen JR. Modified Woodward procedure for Sprengel deformity of the shoulder: Long-term results. Journal of Pediatric Orthopaedics. 1996;16(4):508-513.

7. Khairouni A, Bensahel H, Csukonyi Z, Desgrippes Y,
Pennecot G-F. Congenital high scapula. Journal of Pediatric Orthopaedics B. 2002;11(1):85-88.

8. Ahmad AA. Surgical correction of severe Sprengel deformity to allow greater postoperative range of shoulder abduction. Journal of Pediatric Orthopaedics. 2010;30(6):575-581.

9. Wu S-J, Chin L-S, Kuo J-R. Clinical experience of the Woodward procedure in Sprengel's deformity. Formos $J$ Surg. 2010;43:81-86.

10. Siu K-K, Ko J-Y, Huang C-C, Wang F-S, Chen J-M, Wong T. Woodward procedure improves shoulder function in Sprengel deformity. Chang Gung Med J. 2011;34(4):403-409.

11. Pellegrin K, Kolkman KA, Waldemar Kollig E. Sprengel deformity presenting as a post-traumatic injury in an Afghan boy: A case report. Military Medicine. 2013;178(12):e1379-e1383.

12. Wilkinson J, Campbell D. Scapular osteotomy for Sprengel's shoulder. The Journal of Bone and Joint Surgery British Volume. 1980;62(4):486-490.

13. McMurtry I, Bennet G, Bradish C. Osteotomy for congenital elevation of the scapula (Sprengel's deformity). The Journal of Bone and Joint Surgery British Volume. 2005;87(7):986-989.

14. de Groot JH, van Woensel W, van der Helm FC. Effect of different arm loads on the position of the scapula in abduction postures. Clinical Biomechanics. 1999;14(5): 309-314.

15. Kibler WB. Role of the scapula in the overhead throwing motion. Contemp Orthop. 1991;22:525-532.

16. Myers JB, Laudner KG, Pasquale MR, Bradley JP, Lephart SM. Scapular position and orientation in throwing athletes. The American Journal of Sports Medicine. 2005;33(2):263-271.

17. Pascoal AG, van der Helm FF, Correia PP, Carita I. Effects of different arm external loads on the scapulohumeral rhythm. Clinical Biomechanics. 2000;15:S21S24.

18. Sahrmann S. Diagnosis and treatment of movement impairment syndromes. Elsevier Health Sciences; 2002.

19. Lin JJ, Lim HK, Soto-quijano DA, et al. Altered patterns of muscle activation during performance of four functional tasks in patients with shoulder disorders: Interpretation from voluntary response index. Journal of Electromyography and Kinesiology. 2006;16(5):458-468.

20. Ludewig PM, Cook TM. Alterations in shoulder kinematics and associated muscle activity in people with symptoms of shoulder impingement. Physical Therapy. 2000;80(3):276-291.

21. Kim H, Hwang U, Jung S, Ahn S, Kim J, Kwon O. 
Comparison of shoulder strength in males with and without myofascial trigger points in the upper trapezius. Clinical Biomechanics. 2017;49:134-138.

22. Joung H, Yi C, Jeon H, Hwang U, Kwon O. Effects of 4-week self cross body stretching with scapular stabilization on shoulder motions and horizontal adductor strength in subjects with limited shoulder horizontal adduction. J Sports Med Phys Fitness. 2018;May 29 [Epub ahead of print].

23. Park K-n, Ha S-m, Kim S-h, Kwon O-y. Immediate effects of upper trapezius stretching in more and less tensed positions on the range of neck rotation in patients with unilateral neck pain. Physical Therapy Korea. 2013;20(1):47-54.

24. Hwang U-J, Kwon O-Y, Yi C-H, Jeon H-S, Weon J-H, Ha S-M. Predictors of upper trapezius pain with myofascial trigger points in food service workers: The STROBE study. Medicine. 2017;96(26):e7252.

25. Ha S-m, Kwon O-y, Cynn H-s, et al. Comparison of electromyographic activity of the lower trapezius and serratus anterior muscle in different arm-lifting scapular posterior tilt exercises. Physical Therapy in Sport. 2012;13(4):227-232.

26. Fragala-Pinkham MA, Haley SM, Rabin J, Kharasch VS. A fitness program for children with disabilities. Physical Therapy. 2005;85(11):1182-1200.

27. Reed D, Cathers I, Halaki M, Ginn KA. Does changing the plane of abduction influence shoulder muscle recruitment patterns in healthy individuals? Manual Therapy. 2016;21:63-68.

28. Forte FC, de Castro MP, de Toledo JM, Ribeiro DC, Loss JF. Scapular kinematics and scapulohumeral rhythm during resisted shoulder abduction-Implications for clinical practice. Physical Therapy in Sport. 2009;10(3):105-111.

29. Ludewig PM, Hoff MS, Osowski EE, Meschke SA, Rundquist PJ. Relative balance of serratus anterior and upper trapezius muscle activity during push-up exercises. The American Journal of Sports Medicine. 2004;32(2):484-493.

30. Ebaugh DD, McClure PW, Karduna AR. Threedimensional scapulothoracic motion during active and passive arm elevation. Clinical Biomechanics. 2005; 20(7):700-709.

31. Janda V. Muscles and motor control in cervicogenic disorders: assessment and management. Physical therapy of the cervical and thoracic spine. Edinburgh: Churchill Livingstone; 1994:195-216.

32. Cools AM, Witvrouw EE, Declercq GA, Danneels LA, Cambier DC. Scapular muscle recruitment patterns: Trapezius muscle latency with and without impingement symptoms. The American Journal of Sports Medicine. 2003;31(4):542-549. 